$\mathbb{T}$ periodica polytechnica

Electrical Engineering and Computer Science

57/1 (2013) 19,33

doi: 10.3311/PPee.2079

http://periodicapolytechnica.org/ee

Creative Commons Attribution (i)

RESEARCH ARTICLE

\section{An X-ray CAD system with ribcage suppression for improved detection of lung lesions}

\author{
Áron Horváth / Gergely Orbán / Ákos Horváth / Gábor Horváth
}

Received 2013-04-02, accepted 2013-06-25

\begin{abstract}
The purpose of our study is to prove that eliminating bone shadows from chest radiographs can greatly improve the accuracy of automated lesion detection. To free images from rib and clavicle shadows, they are first segmented using a dynamic programming approach. The segmented shadows are eliminated in difference space. The cleaned images are processed by a hybrid lesion detector based on gradient convergence, contrast and intensity statistics. False findings are eliminated by a Support Vector Machine. Our method can eliminate approximately $80 \%$ of bone shadows (84\% for posterior part) with an average segmentation error of $1 \mathrm{~mm}$. With shadow removal the number of false findings dropped from 2.94 to 1.23 at $63 \%$ of sensitivity for cancerous tumors. The output of the improved system showed much less dependence on bone shadows. Our findings show that putting emphasis on bone shadow elimination can lead to great benefits for computer aided detection.
\end{abstract}

\section{Keywords}

$C A D \cdot C A D e \cdot$ chest radiograph · bone shadow elimination . anatomical segmentation · lung nodule detection · lung cancer

\section{Acknowledgement}

The authors express their thanks to all physicians of Pulmonological Clinic, Semmelweis University, who helped us with validated images and to the development staff of ImSoft ltd. This work was partly supported by the grants TÁMOP - 4.2.2.B-10/12010-0009 and KMR_12-1-2012-0122.

\section{Áron Horváth}

Department of Measurement and Information Systems, Budapest University of Technology and Economics, Magyar tudósok krt. 2., H-1117 Budapest, Hungary e-mail: archee@mit.bme.hu

\section{Gergely Orbán}

Department of Measurement and Information Systems, Budapest University of Technology and Economics, Magyar tudósok krt. 2., H-1117 Budapest, Hungary

\section{Ákos Horváth}

Innomed Medical Zrt., Szabó József u. 12., H-1146 Budapest, Hungary

\section{Gábor Horváth}

Department of Measurement and Information Systems, Budapest University of Technology and Economics, Magyar tudósok krt. 2., H-1117 Budapest, Hungary

\section{Introduction}

Lung cancer is one of the most concerning health problems of the developed world. Extremely high mortality makes it the most common cause of cancer death. It was recently shown that mortality can be reduced with an early diagnosis [1] but it should be done in a mostly symptomless stage of the disease. Therefore screening has the potential to increase the success rate of the treatment. A screening method has to be affordable and side effect-free while being able to detect most lesions, in other words to have high sensitivity. One of the possible methods for screening is chest radiography as it is widespread, cheap and poses the subject to only a low radiation dose. Its main disadvantage is the moderate sensitivity. According to previous studies, $30 \%$ of lung nodules can be overlooked on chest radiographs [2]. Other results have shown that sensitivity can be improved by using a CADe system [3-5].

An extensive amount of CADe systems have already been published for the problem of lung nodule detection. Only a few of the recently published ones are [6-12]. A brief description of them can be found in [13]. The most important problem of existing CADe systems is the low positive predictive value. In other words high sensitivity can only be reached at the cost of many false detections. Most of these published systems can detect 60$70 \%$ of cancerous tumours, while they also mark roughly four false positive regions on each image. This detection capability allows them to be used only as a second reader. Usability of CADe systems can be improved either by reducing the number of false detections - to give the examiner less extra work -, or by finding more true lesions - to increase sensitivity. The detections of CADe should be also complementary to the findings of radiologists, to better improve sensitivity when radiologists and CADe work in cooperation.

Low detection capability on chest radiographs is partly due to overlapping anatomical structures like shadows of bones. The presence of the ribcage and the clavicles on the radiographs can cause two common detection errors. They may conceal the shadows of abnormalities by darkening the image thus reducing contrast resulting in false negative cases. On the other hand, rib crossings on the radiographs sometimes mimic con- 
vex structures appearing to be real lesions. This can introduce false positive findings. Both of the two effects seriously affect human examiners and CADe systems too; however, the hiding effect is problematic mostly to radiologists and physicians while the CADe system suffers rather from false positives due to rib crossings. We claim that properly compensating bone shadows has a great potential to reduce the number of a CADe system's false findings.

Two main approaches are known for bone shadow removal. The first one is a hardware approach eliminating shadows based on two images taken. It is called dual-energy subtraction radiography (DESR) and requires special hardware [14]. This method is not discussed in this paper. The other approach does only software post-processing thus it works well together with conventional X-ray machines.

There were several previous attempts to eliminate bone shadows by software, although the problem is far from being solved. Two main approaches exist. The first one directly suppresses bone shadows by applying a - usually learning - filter, like in [15] and [16]. This requires training samples from DESR methods; however, unsupervised methods also exist, like [17].

The second approach decomposes the problem into segmenting the bone shadows followed by the removal itself. Segmentation in its simplest form involves fitting of a ribcage model to the input image. [18] used a parabolic model and least squares fitting. [19] preferred a mixed second order model and utilized the Hough transform for fitting. It is one of the few solutions targeting also at the anterior part of ribs as most solutions only segment the more visible posterior part. [20] was the first to restrict the search to the viewable lung field and to use vertical intensity profiles for the search. They involved rule-based methods and introduced a refinement step after fitting the model. [21] was the first to utilize dynamic programming for fitting vertical profiles. Their refinement method used active contours. [22] created a statistical model for the whole ribcage described by 10 parameters. [23] proposed to fit a point distribution model. [24] suggested a pixel-wise classification method very different from previous approaches.

Bone shadow removal after segmentation usually involves the estimation of the bone intensity profile based on the contrast of the previously determined edges and then the subtraction of this estimated profile, like implemented in [25] and [26]. A refinement of this method is used in [27].

Recent studies confirmed that bone shadow suppression can help human examiners in finding lung nodules [28-30]. Although [31] recently compared the effect of different bone shadow elimination techniques to a commercially available CADe system, they took a different approach. They did not involve a solution completely omitting bone shadow removal as the CADe system they used with unprocessed images suppressed the bones at a later point in the process. Therefore their method was not able to measure the general usefulness of bone shadow removal. They also did not re-train the CADe system for the different kind of images which is a fundamental step in our approach.

We expected that cleaning the images helps our existing CADe scheme to produce fewer false positive findings. As the area originally hidden by bone shadows cannot be perfectly restored based on a single radiograph, we also created features helping a supervised classifier to eliminate falsely detected structures caused by bones. In the sequel, we describe our existing CADe scheme, the bone shadow removal algorithm and the modifications to improve detection. Afterwards, we demonstrate the results and compare the system to the one without shadow removal.

\section{Materials and Methods}

\subsection{Overview}

The main goal of our CADe system is to find as many lesions as possible on chest radiographs with the lowest number of false findings. The targeted lesions are primarily lung nodules appearing as approximately round shaped dark shadows. These objects are often signs of cancerous tumors. We also try to find infiltrated areas. These are irregularly shaped shadows with less definite border and can also be signs of lung cancer or other interstitial lung diseases (ILD), often tuberculosis (TB).

Our method for finding lung lesions can be separated into three major steps: image segmentation, image filtering and false positive reduction. Figure 1 shows an overview. First, the anatomical structures of the chest are segmented. This involves separating the viewable lung field and the segmentation of bone shadows including the clavicle and the full ribcage. There are several satisfactory solutions for the problem of lung field segmentation [24, 32]. Our own algorithm delineates not only the outline of the lung fields, but provides the full boundary of the ribcage as well, as described in [33]. The viewable area is used to assist ribcage segmentation and to reduce the possible set of locations for lesions, as we only target the ones partly or completely inside this area.

The method we use for the segmentation of the clavicles is discussed in [34]. The ribcage segmentation algorithm is based on [35] and some details are discussed in [36]. Here we only provide an overview and a detailed evaluation of ribcage segmentation.

In the second major step various image filters are run to enhance the visibility of lung lesions. The first filter uses the result of bone segmentation to suppress their shadows. Then three different filters are run in parallel to enhance different types of lung lesions. A Constrained Sliding Band Filter (CSBF) is utilized to detect small and circular lung nodules. A method referenced as Large Nodule Filter (LNF) is responsible for enhancing large and also circular nodules. Last, an Outlier Area Filter (OAF) identifies and segments amorphous but high contrast suspicious areas. The enhanced regions of the image are then extracted by thresholding and lesion borders are determined.

In the last step a large fraction of false findings are removed 


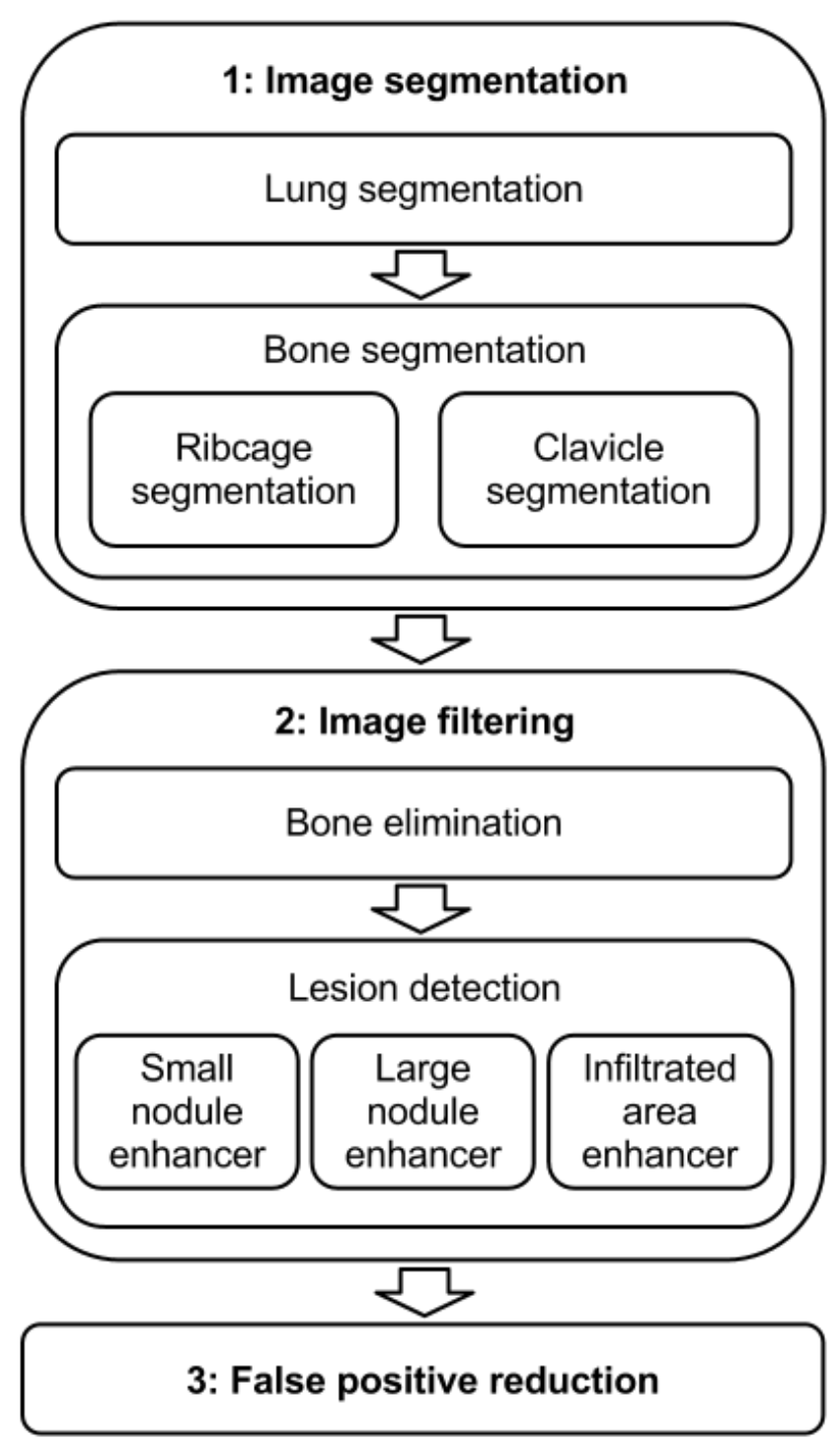

Fig. 1. The main steps of the radiograph analysis.

by supervised learning methods. A hierarchy of SVM-s classifies the candidates based on many texture and geometry features.

\subsection{Ribcage segmentation}

Looking at chest X-ray images an apparent regularity can be observed at first glance: the ribs have a quasi parallel arrangement. So it seems feasible to model the ribcage somehow. Even though the two sides of the ribcage appear to be symmetric, it is hard to select properties of this symmetry which stand for all the instances of a wider image set, where occasional disorders and irregularities may occur. Therefore we handle the two sides of the lungs independently.

To build a complete model - as in [23] and [22] - which is capable of enumerating the ribs one-by-one and to specify their positions would require too many parameters and still would not be accurate enough to handle all the anomalies that may occur in a wider image set. The elimination requires high accuracy, thus we chose a different approach. We restrict our model only to the slopes of ribs. Our model assigns a slope to every point of the area of the bounding box of a lung field, this way it neglects the position information of the ribs. These slopes can be described by a two dimensional function. Previously we investigated the applicability of analytical functions like rational polynomials [35], but application of a simple smooth slope field map resulted in better overall performance. The optimal slope field is searched around an average slope field by fitting small arcs to the image gradients.

This slope field can be applied to align and rescale vertical intensity profiles. This approach goes back to the early times of ribcage segmentation [20]. By aligning these profiles we get a simple one dimensional signal on which local maxima and minima selection gives the vertical positions of the upper and lower borders of ribs. This selection process is by no means straightforward, because there can be much more extrema than needed. To tackle this problem we incorporated various correlations between the position of a rib border and the distances between this and its neighbouring rib borders. This statistics was then formulated into a dynamic programming problem, which provides the positions of the rib borders. From these positions approximate rib borders can be generated based on the aforementioned slope field. The final rib borders are then obtained by refining these by a special dynamic programming based active contour algorithm. The main steps can be followed in figure 2

The main advantage of using a slope field over a global shape model is, that the slope field is almost independent of the rib thickness and spacing information of the ribcage, therefore the search for the complete ribcage can be divided into two distinct subproblem, which reduces the complexity of the overall search.

This solution works well for the posterior part of the ribs, which is more visible on chest radiographs, but for the anterior part we applied a different procedure. It launches a set of spiral curves from the ribcage boundary and iteratively fits them to the image contours by a dynamic programming active contour algorithm. This finds the upper borders, while the lower borders are attached by a statistical point distribution model, and the final borders are got after the same refinement step used for the posterior parts. The details of the whole complex ribcage segmentation algorithm is written in a forthcoming publication.

\subsection{Bone elimination}

The segmentation data is used to remove the bone shadows from the images in order to enhance the visibility of the lung structure. The elimination is based on Simko's work [27]. He smoothed the bone borders on vertically differentiated images, then subtracted the smoothed areas from the original differentiated image. After an integration step he got the bone shadow free image. It worked quite fair on the clavicle, but close to the lateral parts of the ribs it fails. To overcome this we differentiate perpendicular to the bones' mid-line. 


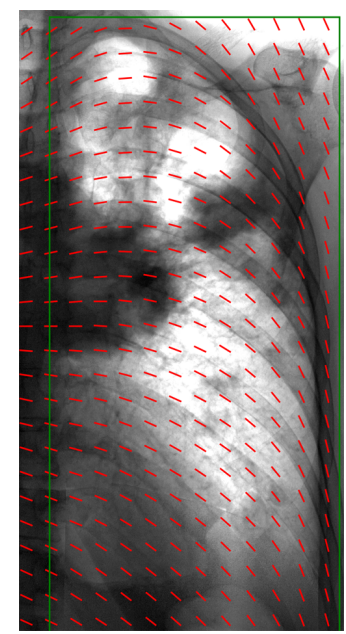

a. Average slope field

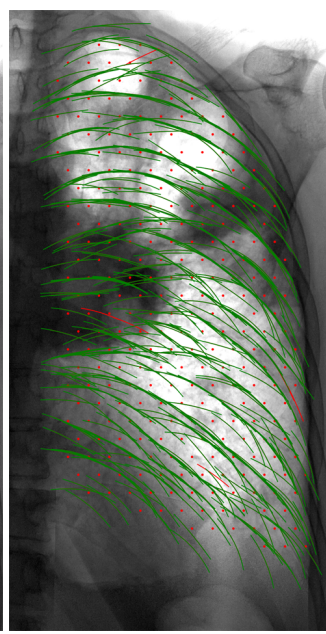

b. Fitted arcs

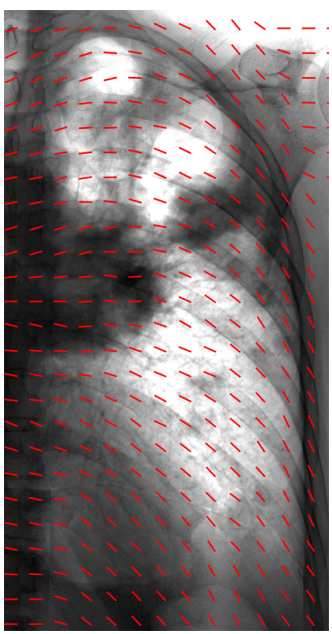

c. Fitted slope field

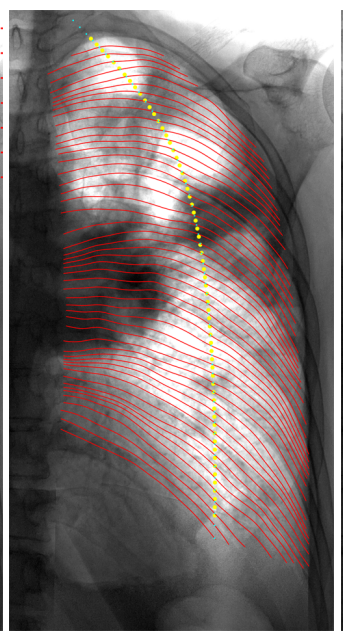

d. Possible curves

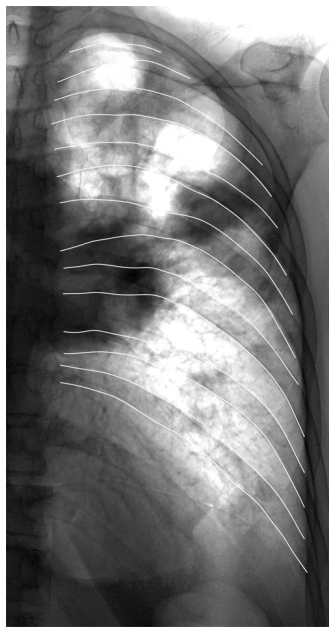

e. Selected curves
Fig. 2. Overview of posterior ribcage segmentation. Arcs are fitted to the image to fine tune an average slope field, then some of the curves generated from this slope field are selected. (The final refined rib borders are not included.

\subsection{Lesion detection}

The main steps of lesion detection are preprocessing, lesion enhancement by the CSBF, LNF and OAF filters, thresholding and lesion segmentation. In the sequel we give a brief description of these methods, while a more detailed presentation can be found in [13].

In the preprocessing step the bone shadow eliminated images are first subsampled to 512 lines of height while keeping the aspect ratio to reduce running time. Then, to spread pixel intensities in $[0,1]$, the median value of the pixels in the segmented lung field and the deviation from the median is set to empirical values of 0.35 and 0.18 respectively by scaling and translation of the pixel values.

The first nodule enhancer, the CSBF is a gradient convergence based filter, also a member of the Convergence Index filter family. It is our slight modification of the Sliding Band Filter described in [37]. It is mainly capable of enhancing small and circular structures. In our current setting it aims at structures with a diameter between $5 \mathrm{~mm}$ and $30 \mathrm{~mm}$. The algorithm first generates image gradients with a Sobel filter, then maps the gradient vector lengths by a bounded ramp function $r$.

$$
r(x, a, b)=\left\{\begin{array}{ll}
0 & x<a \\
x-a & a \leq x<b \\
b-a & b \leq x
\end{array},\right.
$$

where $x$ is the original vector length, $a$ and $b$ are parameters. In our case $a$ is set to 0 and $b$ is set to 0.0025 , a length above which the gradient vector is unlikely to be noise. After the mapping the CSBF filter output can be calculated as follows.

$$
\begin{aligned}
C S B F(x, y) & =\max _{R_{\min } \leq r \leq \frac{R_{\max }}{c}} \frac{1}{N} \sum_{i=1}^{N} \operatorname{Cmax}_{i r}, \\
\text { Cmax }_{i r} & =\max _{r \leq n \leq r * c} \frac{1}{d} \sum_{m=n}^{n+d} \cos \theta_{i m} r\left(\left|g_{m, i}\right|, a, b\right),
\end{aligned}
$$

The difference between Fig. 2/c. and Fig. 2/3a. is difficult to notice, but it is significant for the further steps. )

where $R_{\min }, R_{\text {max }}$ are the bounds of the target object radius, $c$ is the shape constraint coefficient, $N$ is the number of radial directions, $d$ is the width of the band, $g_{m, i}$ is the $m^{\text {th }}$ gradient vector along the $i^{\text {th }}$ radial direction, $\theta_{i m}$ is the angle of $g_{m, i}$ and the corresponding radial vector and $a$ and $b$ are the same as above. $R_{\text {min }}$ and $R_{\max }$ were set to match the smallest and larges nodules to be found. $c$ controls the maximum distortion from a perfectly circular shape and found out to be optimal around 1.2. $N$ was set to 16 , providing a good compromise between precision and speed. The parameter $d$ affects noise sensitivity and was set to $5.6 \mathrm{~mm}$. An example result can be seen in Figure 3 .

We created the LNF to enhance nodules with a diameter between $30 \mathrm{~mm}$ and $75 \mathrm{~mm}$ and high contrast, while letting them to lie almost completely outside of the viewable lung field. The latter feature enables it to find nodules mostly under the shadow of the heart or the diaphragm. The algorithm first performs local contrast enhancement (LCE) [38], but taking into account the border of the viewable lung field. The output - $G(x, y)-$ is given by the following equations.

$$
\begin{aligned}
& G(x, y)=\frac{1}{1-\exp \left[-\left(F(x, y)-\frac{1}{|R(x, y)|} \sum_{(u, v) \in R(x, y)} F(u, v)\right)\right]} \\
& R(x, y)= \\
& = \begin{cases}\left\{(u, v) \mid(u-x)^{2}+(v-y)^{2}<2 r^{2}\right\} \cap L & (x, y) \in L \\
\left\{(u, v) \mid(u-x)^{2}+(v-y)^{2}<2 r^{2}\right\} \cap / L & \text { otherwise }\end{cases}
\end{aligned}
$$

where $F$ is the original image, $L$ is the viewable lung field and $r$ is the radius of the targeted nodule. The trimming of $R$ with $L$ ensures we have a homogeneous area completely inside or outside the lung as the contrast in these two regions can be much different. The rationale behind using the logistic function is to get a result in between local normalization and local thresholding.

The second and last step of LNF is a top-hat filtering, which is a simple convolution by a cylinder shaped kernel with radius $r$. In our implementation the side of the cylinder is slightly tilted, 
so we get a cut-cone shaped kernel rather than a cylinder. This reduces the noise sensitivity of the method and also better enhances not perfectly circular objects. The LNF algorithm is run in multi-scale with four different values of $r$ to find nodules of different size. A typical positive result of the LNF can be seen in Figure 3 b.

The filter created for detecting amorphous structures is best described as an intensity based novelty detector. Based on a training set of healthy patients it learns the distribution of the pixel values for each coordinate in the lung where the coordinate system is described later. An input image is first processed by a median filter to eliminate pixel size noise. Then every pixel with an unprobable value - darker than $97 \%$ of the training samples for the given location is marked. The last step applies morphological opening and discards small hits close to the lung border. This is to eliminate false results caused by small structures and lung segmentation errors. The remaining outlying regions are returned as suspicious areas. An example result of the OAF can be seen in Figure 3 .

To describe the coordinates for the OAF we created a system called lung polar coordinate system. The origin $(O)$ is defined as the center of gravity of the lung masks marking the viewable lung areas. For a given point $p$ the first coordinate $\varphi$ is the angle of the vector $\overrightarrow{O p}$ with the vertical axis, mapped to $[-\pi, \pi]$. The second coordinate is a normalized distance $d(p, \varphi)$ depending on the location and the first coordinate. Considering $e_{p}$ the half-line starting from the origin and crossing $p, d$ is the following.

$$
d(p, \varphi)=\frac{\left\|p-I_{1 \varphi}\right\|}{\left\|I_{2 \varphi}-I_{1 \varphi}\right\|},
$$

where $I_{1}$ is the closest while $I_{2}$ is the farthest intersection of $e_{p}$ with the lung mask, $\|x\|$ denoting the Euclidean distance. $\varphi$ and $d$ are quantized to 200-200 discrete values.

After the three lesion enhancer filters have been run the outputs are thresholded to get the lesion candidates. For the CSBF a dynamic threshold is used which is set to produce 35 candidates for each image. The LNF output is thresholded by a constant which typically produces two to three candidates per image. From the OAF output all the marked regions are collected yielding on average one result per image.

The segmentation of the lesion candidates is different for each enhancer output. The OAF generates area borders as a side product as connected regions will be candidates. For the CSBF and the LNF nodule segmentation is done by an optimization algorithm with preference towards circular shapes while adjusting the border to high contrast discontinuities. The optimization is done using dynamic programming.

\subsection{False positive elimination}

To reduce the number of false candidates generated in the lesion detection step a Support Vector Machine classifier (SVM or SVC) is utilized [39]. The efficient application of an SVM requires good quality training data, a careful choice of kernel function, an appropriate input vector, and hyperparameter tuning of the generalization coefficient (often denoted by $C$ ) and the kernel parameters.

Our training data comes from a radiograph database of 282 images described later. Positive samples of lesions are both the validated findings of pulmonologists and radiologists, and also the candidates found by the lesion detector close enough to the validated findings. Negative samples are candidates found only on healthy images. On positive images, the candidates that are far from the validated findings are not used for training. This was necessary as physicians tend to mark only some of the lung nodules when the image contains too many of them. Keeping negative samples only from healthy images improved classifier performance on independent test data. Exact thresholds used for training data generation are described in [13]. As 30 times more negative samples were available than positive ones, we used a cost-sensitive version of the SVM. Balancing is achieved by using different $C$ values for positive and for negative samples.

As for the kernel, the isotropic Gaussian function was chosen for the SVM. The input vector of the kernel consists of various features describing texture, geometry and location. The final set of features described in section 2.5.1 is a result of a simple forward selection algorithm selecting from 168 implemented features. As the relevant features turned out to be different for the output of the three nodule enhancing algorithms, separate classifiers are used for each result set. This improves classification performance due to fewer irrelevant features and also reduces training time.

The two hyperparameters of the resulting SVM $-C$ and the width parameter $(\sigma)$ of the kernel - are tuned by an iteratively refining grid search. Cross-validation was used on image level to prevent overfitting.

\subsubsection{The features for classification}

The aforementioned feature selection algorithm identified 27 relevant features out of the total 168 we implemented. The short description of these features is the following.

- Mean and maximum of the average fraction under the minimum (AFUM) filter inside nodule border [40]..$^{1,2}$

- The linearity of nodule border transition. This considers the intensity profiles perpendicular to the nodule border and calculates the error to the best fitting linear approximation. ${ }^{1}$

- The contrast (average intensity fraction) close to the determined nodule border. ${ }^{1}$

- The contrast between the nodule parts inside and outside the viewable lung field. ${ }^{1}$

- Difference of minimum values inside the nodule and in the surroundings of the nodule normalized by the intensity range in the lung field. ${ }^{1}$

- Ratio of nodule and lung field mean intensity. ${ }^{2}$ 


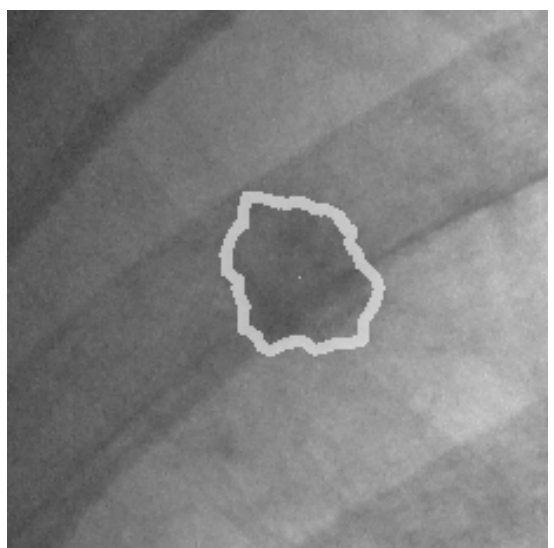

a. A CSBF finding

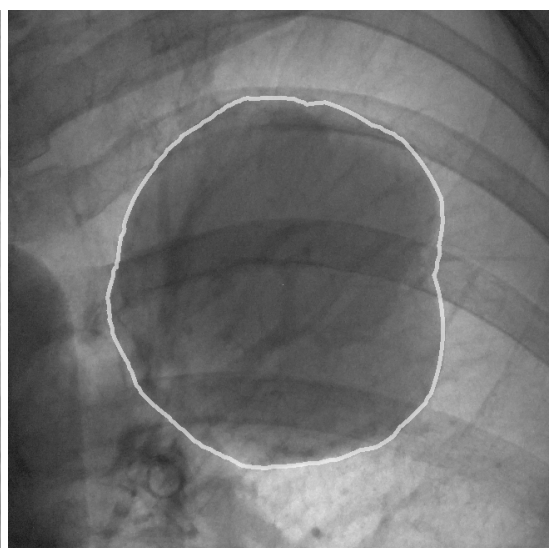

b. A LNF finding

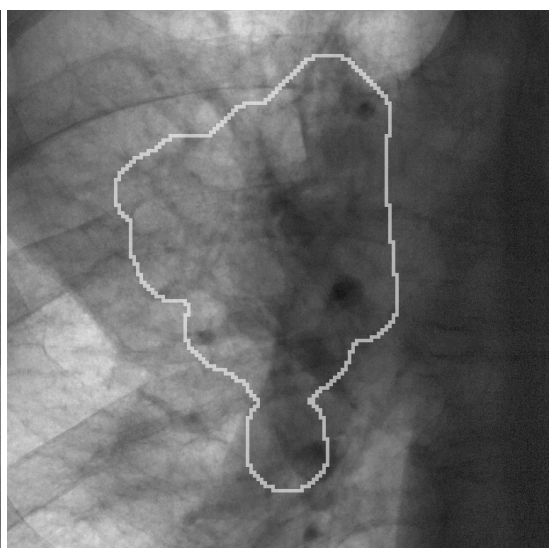

C. An OAF finding.

Fig. 3. Typical findings of the three lesion detectors. From left to right : a. A CSBF finding., b. A LNF finding., c. An OAF finding.

- Difference of nodule and lung field intensity mean. ${ }^{2}$

- Horizontal coordinate measured from the closest point of the lung field to the spine. ${ }^{1,2}$

- Vertical coordinate measured from the topmost point of the viewable lung field. ${ }^{1}$

- LPCS distance coordinate described in section $2.4^{1,2}$

- Standard deviation of a derivative of Gaussian filter output in different directions [9]. The width parameter of the Gaussian function $\left(\sigma_{G}\right)$ was $3 \mathrm{~mm}$. $^{1}$

- Mean of a Laplacian of Gaussian (LoG) filter for the center of the nodule $\left(\sigma_{G}=1.5 \mathrm{~mm}, 6 \mathrm{~mm}\right)[9] .{ }^{1,2}$

- LoG filter value at nodule center $\left(\sigma_{G} \in 3 \mathrm{~mm}, 6 \mathrm{~mm}\right) .{ }^{1}$

- LoG filter mean value inside nodule border $\left(\sigma_{G} \in\right.$ $1.5 \mathrm{~mm}, 3 \mathrm{~mm}, 6 \mathrm{~mm}){ }^{1}$

- LoG filter minimum value inside nodule border $\left(\sigma_{G} \in\right.$ $1.5 \mathrm{~mm}, 6 \mathrm{~mm}){ }^{1}$

- LoG filter standard deviation inside nodule border $\left(\sigma_{G}=\right.$ $3 \mathrm{~mm})^{1}$

- The rank of the candidate in a list sorted by enhancer output intensity. ${ }^{1}$

- Output of the LNF enhancer using the nodule border as a kernel for the top-hat filter instead of the original round shaped one. ${ }^{1}$

- Nodule enhanced image (LNF output) value. ${ }^{2}$

- Average of contrast. Described by [41] as the 2nd feature (and referenced as $\mathrm{H} 2)^{2}$

- Average of difference variance (H10). ${ }^{2}$

- Average of an information measure of correlation (H13). ${ }^{2}$

- Average of sum entropy (H8). ${ }^{2}$

- Joint entropy version one (H12-H13). ${ }^{2}$
- Joint entropy version two (H12-H13). ${ }^{2}$

- Robustness of nodule border. It restarts nodule segmentation from centers near the original one and compares the resulting borders. $^{2}$

- Similarity of histograms inside and outside nodule, near the border. Similarity is simply the number of bins for which the difference between inside and outside is greater than a predefined value. $^{2}$

In the list ${ }^{1}$ and ${ }^{2}$ means that the feature is used for the candidates of the CSBF and LNF respectively. For the OAF the feature selection could not be run due to the small number of positive samples. As the findings of the LNF and OAF have some similar properties, we decided to use the same feature set for both algorithms (except for the "robustness of nodule border" feature as the OAF has a different segmentation method).

To better exploit the results of bone segmentation, three new features were created based on the following observations. First, density variations in bone structure can appear as intensity extrema and may produce false positive findings. Second, areas where bones overlap on the 2 dimensional summation images appear as dark, approximately rhomboid shaped structures frequently recognized by the system as lesions. Third, in the cases where bone shadows cause false positive candidates, the border of the candidate follows the edge of the bone in a relatively large portion. These three observations motivated the following three features respectively.

The first feature calculates the area fraction of the nodule overlapping with a bone structure. The feature can be described formally as

$$
\text { BoneOverlap }_{i}=\frac{\left|\left\{(x, y) \mid(x, y) \in C_{i} \cap\left(\bigcup_{j} B_{j}\right)\right\}\right|}{\left|\left\{(x, y) \mid(x, y) \in C_{i}\right\}\right|},
$$

where $C_{i}$ contains the points of the $i^{\text {th }}$ candidate while $B_{j}$ means the set of points inside the $j^{\text {th }}$ bone structure. |.| denotes the size of the sets. The second feature calculates the overlap of candidates with bone crossings - the areas where two or more bone 
segments overlap with each other. Using the previous notation, the formula is

$$
\text { BoneXOverlap }_{i}=\frac{\left|\left\{(x, y) \mid(x, y) \in C_{i} \cap\left(\bigcup_{j, k}\left(B_{j} \cap B_{k}\right)\right)\right\}\right|}{\left|\left\{(x, y) \mid(x, y) \in C_{i}\right\}\right|} .
$$

The third feature calculates the fraction of the nodule perimeter that runs near to a bone shadow border. It can be calculated as

$$
\begin{aligned}
& \text { FollowBoneEdge }_{i}= \\
& \qquad=\frac{\left|\left\{(x, y) \mid(x, y) \in \delta C_{i}, \min _{j} d\left((x, y), \delta B_{j}\right)<1.5 \mathrm{~mm}\right\}\right|}{\left|\left\{(x, y) \mid(x, y) \in \delta C_{i}\right\}\right|},
\end{aligned}
$$

where $\delta C_{i}$ and $\delta B_{j}$ are the endpoint set of $C_{i}$ and $B_{j}$, while $d$ is the Euclidean distance.

\subsection{The radiograph database}

We tested the lesion detection on a private chest X-ray database containing images of 407 patients where 259 of the cases contained at least one malignant lung nodule. Nodule diameter ranged from $2 \mathrm{~mm}$ to $98 \mathrm{~mm}$, the average was $23 \mathrm{~mm}$. Most of the malignant cases were validated by CT. The images came from a TOP-X DR series digital X-ray machine by Innomed Medical Zrt. The detector used a maximal resolution of $3000 \times 3000$ pixels with $0.16 \mathrm{~mm}$ pixel size in both dimensions.

For the evaluation of bone segmentation we used a subset of 30 images for which reference bone contours were available from a human observer. Out of these images five were segmented also by another independent observer for the interobserver tests.

For the lesion detector we separated 282 images for training and cross-validated testing and kept the remaining 125 images purely for testing. This way we could generate results for all 407 images without overfitting.

\subsection{Bone segmentation}

Numerous ways of measuring the quality of ribcage segmentation have been applied in the literature. Different measurements tell different things about the results. To make our results comparable to others we had to evaluate them in various ways. First, we show detailed results when using a strict method involving the pairing of found and reference bones. Then we show the results of a more permissive test also used in the literature, which simply uses overlap with minimal pre-processing.

The first test was conducted on a set of 30 images. Previously these images were manually segmented by a human observer. His task was to delineate all the visible ribs. 5 of these images were also segmented by another independent observer. There was no accord between the two observers in how many ribs they have found on each image. The ribs in the abdominal region are often obscured, and some of them are cropped by the image borders.

Our current algorithm segments the anterior and posterior parts of the ribs independently, but the human observers delineated the whole ones. There is a problem with this differ- ence, the Jaccard index $\left(J(A, B)=\frac{|A \cap B|}{|A \cup B|}\right)$ becomes difficult interpret, as the intersection between the areas will always be much smaller than their union, and the perfect solution will not get 100

The rib shadow elimination makes sense mainly above the lung fields, but to make it comparable with some other results in ribcage segmentation [23], [24] we have done two different measurements. First, we simply compared the posterior and anterior parts by their manual counterparts, then we trimmed these by the approximated complete lung field. In contrast to the general concept of visible lung fields we included the area of the heart as well.

Our results can be seen in Figure 4 We ordered the images by their Jaccard index, and selected the worst, the best and the median from the ordered list. We did this ordering separately for the posterior and the anterior parts, and separately for the lung field trimmed and the full results as well.

We did some quantitative measurements as well, which can be seen on table 4 (average values), and on table $1,2,3$ for the worst, median and best images respectively. To make these numbers easier to interpret we included the respective images as well (Figure 4).

The segmentation works independently on the left and right side, so we measured the results separately and the resulting numbers should be interpreted per lung side. The output of our algorithm does not provide a numbering for the ribs, only returns a simple set of ribs. Therefore the evaluation process had to handle the problem of finding the corresponding segmented rib for each reference rib. This pairing was done based on the Jaccard index. Every reference rib got a corresponding segmented rib, even if that segmented rib belonged to more than one reference rib.

We were interested in how many of the 12 ribs our algorithm has found. For this we defined a rib being found when the Jaccard index was above a threshold of 0.55. It may appear to be low, but even the inter-observer results were between 0.61 and 0.84. According to Table 4 our algorithm found on average 5.93 posterior and 3.6 anterior ribs on an image, but when the considered area of both the segmented and the reference bone are restricted to the lung fields the numbers go up to 6.16 and 4.3 respectively. This increase can be explained by the fact, that we got higher Jaccard indices for the restricted area, because some parts of the ribs (both the reference and the segmented) are trimmed by the same curve (lung outline), which reduces the part of outline where the two area can differ.

We call a reference rib missed if there is no corresponding segmented rib which reached the threshold. We call a segmented rib false if there is no reference rib for which the Jaccard index is above the threshold. The average Jaccard index in Table 5 is calculated by simply averaging the values over the ribs, but a smaller missing rib can cause less harm for the elimination than a longer one. To incorporate this effect we introduced a 
Tab. 1. Worst case images of bone segmentation results when CADe compared with observer1. (Fig.6 left)

Tab. 2. Median case images of bone segmentation results when CADe compared with observer1. (Fig.6 middle)

Tab. 3. Best case images of bone segmentation results when CADe compared with observer1. (Fig.6 right)

\begin{tabular}{|c|c|c|c|c|}
\hline & \multicolumn{2}{|c|}{ Posterior } & \multicolumn{2}{|c|}{ Anterior } \\
\hline & inside lung & full image & inside lung & full image \\
\hline Number of Reference Ribs & 8,50 & 10,00 & 8,00 & 10,50 \\
\hline Number of Segmented Ribs & 7,00 & 6,50 & 3,50 & 5,50 \\
\hline Found & 5,00 & 5,50 & 0,50 & 0,00 \\
\hline Missed & 3,50 & 4,50 & 7,50 & 10,50 \\
\hline Found per All & 0,59 & 0,55 & 0,06 & 0,00 \\
\hline False & 2,00 & 1,00 & 3,00 & 5,50 \\
\hline False per Segmented Ribs & 0,27 & 0,14 & 0,50 & 1,00 \\
\hline Average Jaccard Index & 0,49 & 0,41 & 0,10 & 0,09 \\
\hline Weighted Jaccard Index & 0,46 & 0,36 & 0,07 & 0,07 \\
\hline Average Sensitivity & 0,60 & 0,48 & 0,15 & 0,22 \\
\hline \multirow[t]{3}{*}{ Weighted Sensitivity } & 0,67 & 0,46 & 0,13 & 0,16 \\
\hline & \multicolumn{2}{|c|}{ Posterior } & \multicolumn{2}{|c|}{ Anterior } \\
\hline & inside lung & full image & inside lung & full image \\
\hline Number of Reference Ribs & 7,50 & 10,00 & 7,50 & 10,50 \\
\hline Number of Segmented Ribs & 6,00 & 8,00 & 5,50 & 6,50 \\
\hline Found & 6,00 & 6,00 & 4,50 & 1,50 \\
\hline Missed & 1,50 & 4,00 & 3,00 & 9,00 \\
\hline Found per All & 0,79 & 0,60 & 0,59 & 0,15 \\
\hline False & 0,00 & 2,00 & 1,00 & 5,00 \\
\hline False per Segmented Ribs & 0,00 & 0,25 & 0,20 & 0,79 \\
\hline Average Jaccard Index & 0,63 & 0,53 & 0,46 & 0,31 \\
\hline Weighted Jaccard Index & 0,62 & 0,49 & 0,43 & 0,33 \\
\hline Average Sensitivity & 0,72 & 0,61 & 0,60 & 0,37 \\
\hline \multirow[t]{3}{*}{ Weighted Sensitivity } & 0,81 & 0,62 & 0,65 & 0,48 \\
\hline & \multicolumn{2}{|c|}{ Posterior } & \multicolumn{2}{|c|}{ Anterior } \\
\hline & inside lung & full image & inside lung & full image \\
\hline Number of Reference Ribs & 7,00 & 10,00 & 6,50 & 9,00 \\
\hline Number of Segmented Ribs & 7,00 & 8,00 & 7,50 & 8,00 \\
\hline Found & 7,00 & 7,50 & 4,50 & 5,00 \\
\hline Missed & 0,00 & 2,50 & 2,00 & 4,00 \\
\hline Found per All & 1,00 & 0,75 & 0,70 & 0,56 \\
\hline False & 0,00 & 0,50 & 3,00 & 3,00 \\
\hline False per Segmented Ribs & 0,00 & 0,06 & 0,39 & 0,38 \\
\hline Average Jaccard Index & 0,81 & 0,60 & 0,64 & 0,48 \\
\hline Weighted Jaccard Index & 0,82 & 0,63 & 0,67 & 0,48 \\
\hline Average Sensitivity & 0,86 & 0,66 & 0,84 & 0,67 \\
\hline Weighted Sensitivity & 0,88 & 0,72 & 0,83 & 0,67 \\
\hline
\end{tabular}

Weighted Jaccard index, which is the following:

$$
\text { WeightedJaccardIndex }=\frac{\sum_{i}\left|R_{i} \bigcap S_{i}\right|}{\sum_{i}\left|R_{i} \cup S_{i}\right|},
$$

where $R_{i}$ denotes the area of the $\mathrm{i}^{\text {th }}$ reference, and $S_{i}$ denotes the area of the $i^{\text {th }}$ segmented rib. The simple averaged Jaccard index is the following:

$$
\text { AverageJaccardIndex }=\sum_{i} \frac{\left|R_{i} \cap S_{i}\right|}{\left|R_{i} \bigcup S_{i}\right|} .
$$

For the elimination it is important to have an accurate bone outline. One can get relatively high Jaccard indices with low outline accuracy, while if the outline is exactly the same, but the reference rib is longer than the segmented one, the Jaccard index degrades in a fast pace. To overcome this we introduced another measure called Distance of Found. It is calculated by pairing the points of the reference borders by the segmented borders. The points without corresponding pairs are left out, and the average distance between the points are calculated. The pairing is carried out by launching perpendicular half-lines from the points of the midline of the two curves, and finding the intersecting points.

E.g. for the untrimmed posterior ribs we got an average of 5.2 pixels, which means $0.83 \mathrm{~mm}$ in reality. These numbers are close to the ones calculated for the inter-observer study, but it should be noted that these were averaged over only the found ribs. The distribution of these distances are shown in Figure 5 .

Another figure of merit we calculated is the specificity and sensitivity of the bone shadow segmentation based on area overlap. The area overlap is the size of the area that is included 
Tab. 4. Average bone segmentation results when CADe compared with observer1.
Tab. 5. Inter-observer bone segmentation results between observer1 and observer2.

\begin{tabular}{|c|c|c|c|c|}
\hline & \multicolumn{2}{|c|}{ Posterior } & \multicolumn{2}{|c|}{ Anterior } \\
\hline & inside lung & full image & inside lung & full image \\
\hline Number of Reference Ribs & 8,40 & 9,50 & 7,65 & 10,20 \\
\hline Number of Segmented Ribs & 7,15 & 7,53 & 6,10 & 7,18 \\
\hline Found & 6,23 & 5,90 & 4,00 & 3,40 \\
\hline Missed & 2,18 & 3,60 & 3,65 & 6,80 \\
\hline Found per All & 0,74 & 0,62 & 0,52 & 0,34 \\
\hline False & 0,93 & 1,63 & 2,10 & 3,78 \\
\hline False per Segmented Ribs & 0,12 & 0,20 & 0,35 & 0,53 \\
\hline Average Jaccard Index & 0,63 & 0,53 & 0,48 & 0,35 \\
\hline Weighted Jaccard Index & 0,63 & 0,48 & 0,44 & 0,33 \\
\hline Average Sensitivity & 0,72 & 0,64 & 0,59 & 0,51 \\
\hline \multirow[t]{3}{*}{ Weighted Sensitivity } & 0,79 & 0,65 & 0,60 & 0,56 \\
\hline & \multicolumn{2}{|c|}{ Posterior } & \multicolumn{2}{|c|}{ Anterior } \\
\hline & inside lung & full image & inside lung & full image \\
\hline Number of Reference Ribs & 8,55 & 8,95 & 7,95 & 10,25 \\
\hline Number of Segmented Ribs & 8,50 & 8,95 & 7,90 & 10,25 \\
\hline Found & 8,10 & 7,80 & 6,90 & 7,40 \\
\hline Missed & 0,45 & 1,15 & 1,05 & 2,85 \\
\hline Found per All & 0,95 & 0,87 & 0,87 & 0,72 \\
\hline False & 0,40 & 1,15 & 1,00 & 2,85 \\
\hline False per Segmented Ribs & 0,05 & 0,13 & 0,12 & 0,28 \\
\hline Average Jaccard Index & 0,77 & 0,71 & 0,73 & 0,63 \\
\hline Weighted Jaccard Index & 0,78 & 0,70 & 0,73 & 0,69 \\
\hline Average Sensitivity & 0,86 & 0,81 & 0,84 & 0,77 \\
\hline Weighted Sensitivity & 0,87 & 0,82 & 0,85 & 0,82 \\
\hline
\end{tabular}

in both the segmentation under investigation and the reference segmentation. Sensitivity of a method or observer versus the reference observer is the fraction of the overlap and the total area of the reference segmentation, in other words the proportion of correctly identified bone area according to the reference. Specificity is the fraction of the overlap and the total area of the method under investigation. One minus the specificity gives the proportion of false positive bone area. We restricted the whole calculation to the viewable lung field as the ribs below the diaphragm are often barely visible, misleading both the algorithm and the human observers.

Table 6 shows the results of the segmentation tests based on bone overlap. In the first column we compared the CADe results to a reference human observer locating only anterior ribs. In the second test the same human observer was asked to segment the complete ribs including both anterior and posterior parts. The third test involved two independent human observers both segmenting complete ribs. This inter-observer comparison shows the obscurity of the problem and gives an approximate upper bound to the achievable metrics for the CADe algorithm.

Comparing the $80.3 \%$ sensitivity for full ribs with the $94 \%$ of the inter-observer result shows that the algorithm misses around $15 \%$ of the determinable part of the bone which equals to approximately one bone per lung field. Segmentiation of the posterior part is even better with $84.1 \%$ sensitivity. Specificity of $90.3 \%$ ensures that the algorithm doesn't produce much false areas. Furthermore, the usual error here is that the anterior rib seg- mentation continues to follow a bone towards the mediastinum even when it becomes hardly visible or not visible at all for a human observer. In many of these cases the rib can be identified retrospectively, thus these cases are rather the miss of the human observer. Furthermore, we observed that the rib parts where the CADe and observers disagreed are barely visible so these sections hardly disturb lesion detection. Sections with high contrast are segmented with higher accuracy.

Figure 6 shows example results for rib segmentation and elimination for the worst, an average and the best cases. It can be seen that even for the worst segmentation the bone shadow eliminated image is adequate. Bone shadows are hardly visible and other fine details are kept.

It is almost impossible to compare our results with other published numbers as both the test samples and the methodology are different. As an example in [23] the authors published $50 \%$ to $80 \%$ sensitivity for the posterior and $50 \%$ for the anterior part of the ribs. These numbers best correspond to the Weighted Sensitivity row in Table 4, however, only the first 10 ribs were assessed in that study. In [22], where the $2^{\text {nd }}$ to $10^{\text {th }}$ posterior ribs were included, the authors reached an accuracy of 0.787 . Their measurement method was most similar to the one used in the first column of Table 6. Based on these numbers we cannot state that our solution is better or worse, we can only say it performs comparably. 
Fig. 4. Example results of the ribcage segmentation. The worst, the median and the best cases are shown from left to right respectively. The overlapping area is green, the false positive is cyan, the true negative is yellow.
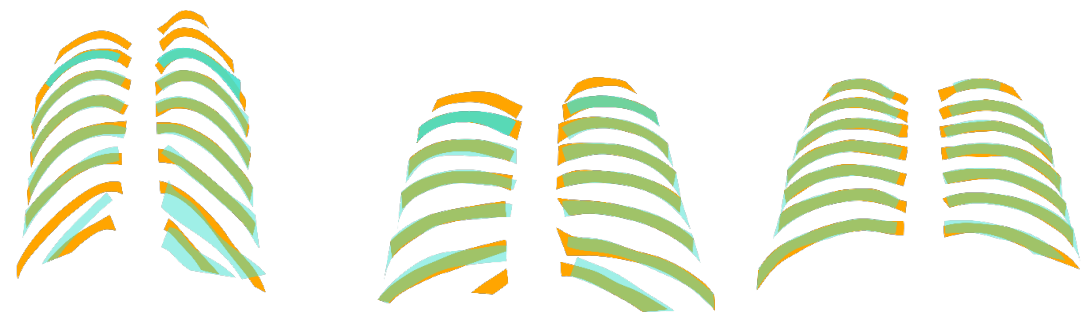

a. Ordered by the Jaccard index for the posterior ribs restricted to the viewable lung field.
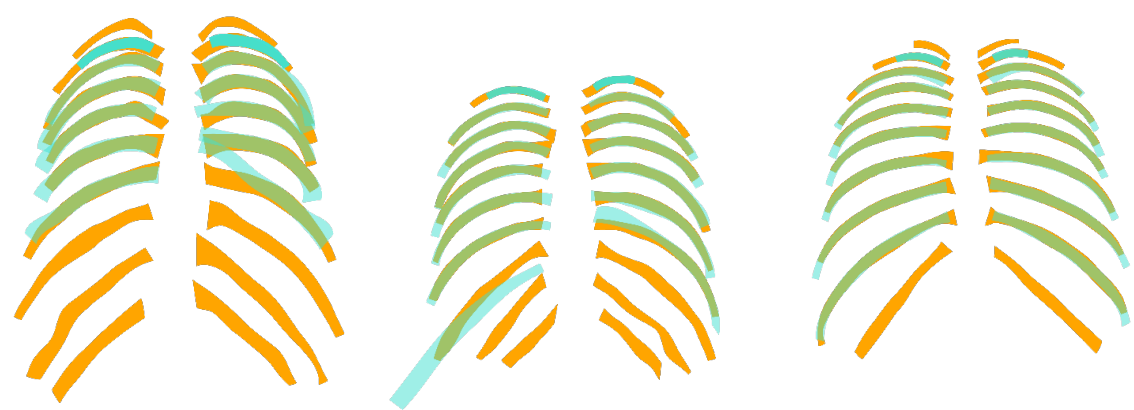

b. Ordered by the Jaccard index for the posterior ribs for the full image.
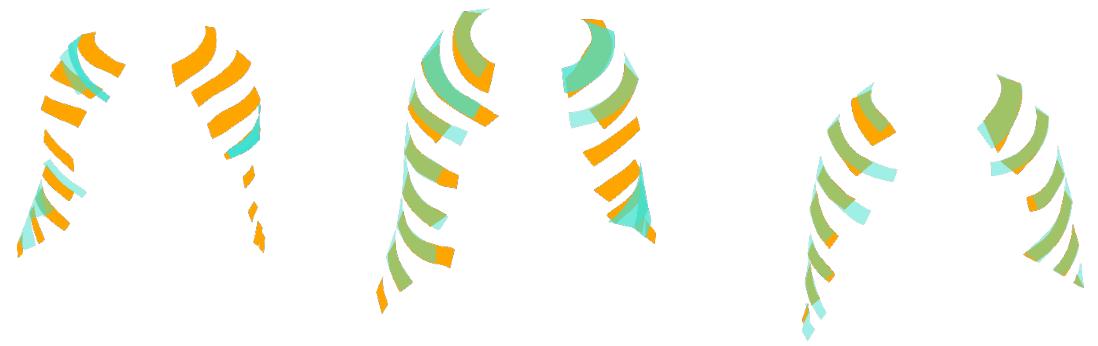

c. Ordered by the Jaccard index for the anterior ribs restricted to the viewable lung field.
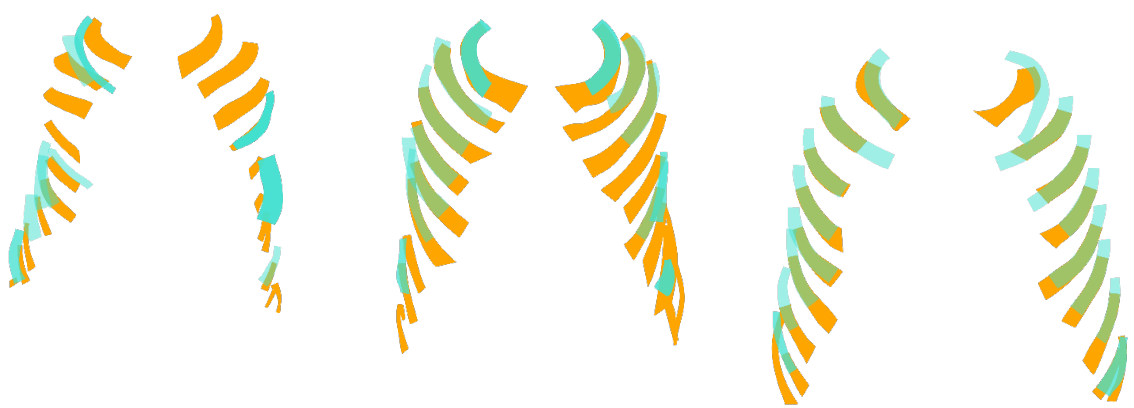

d. Ordered by the Jaccard index for the anterior ribs for the full image.

Tab. 6. Bone locator sensitivity and specificity in $\%$ based on overlap statistics.

\begin{tabular}{rccc}
\hline & $\begin{array}{c}\text { CADe vs Observer1 } \\
\text { posterior ribs }\end{array}$ & $\begin{array}{c}\text { CADe vs Observer1 } \\
\text { complete ribs }\end{array}$ & $\begin{array}{c}\text { Observer2 vs Observer1 } \\
\text { complete ribs }\end{array}$ \\
\hline Sensitivity & 84.1 & 80.3 & 94 \\
Specificity & 87.6 & 90.3 & 94.1 \\
Sample size & 30 & 30 & 5 \\
\hline
\end{tabular}

\subsection{Lesion detection}

For the following measurements 10-fold cross validation with 30 repetitions was used. Results are demonstrated on freeresponse receiver operating characteristic (FROC) curves, showing the sensitivity as a function of average number of false positives produced for each image. On the final output, a CADe drawing was considered to be correct if its centroid - center of gravity - was located inside a physician's marker; otherwise it was labeled as a false positive.

We evaluated our CADe system in three configurations to analyze the effect of bone localization and removal. The first configuration used no bone information at all, the second employed 


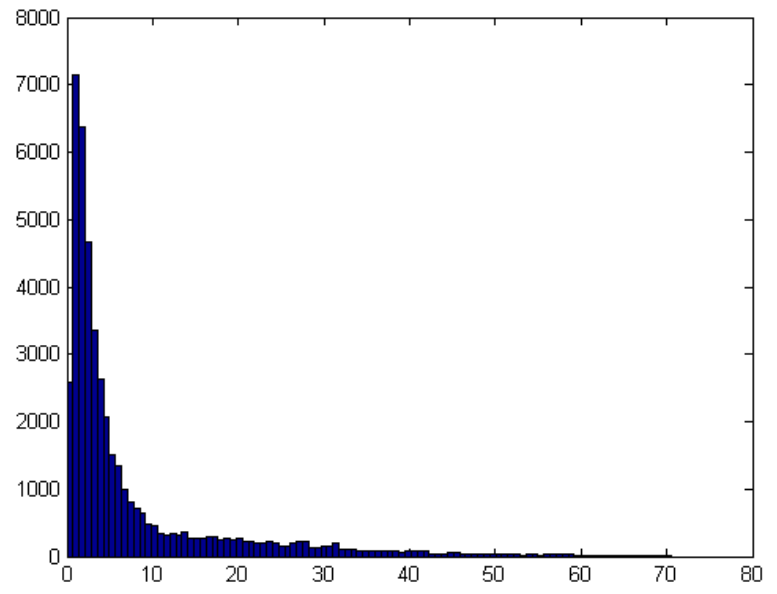

a. Segmented vs observer1 anterior ribs.

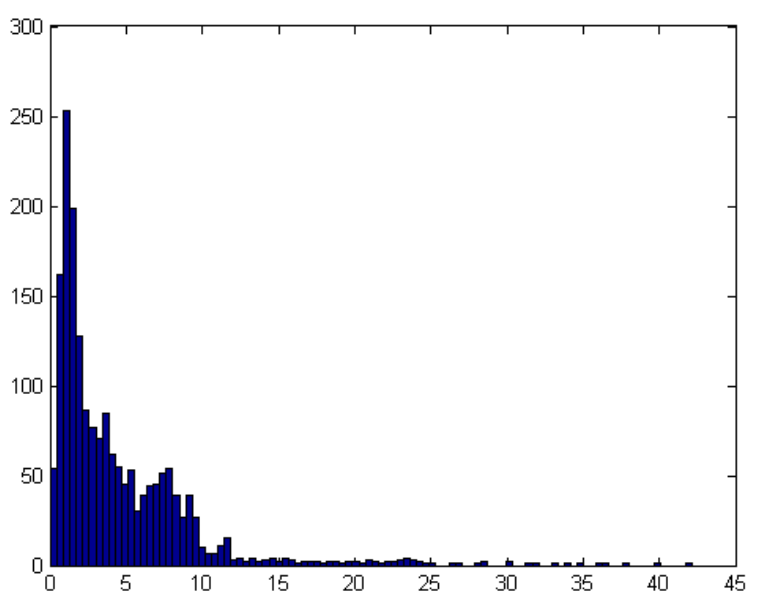

c. Inter-observer anterior ribs.

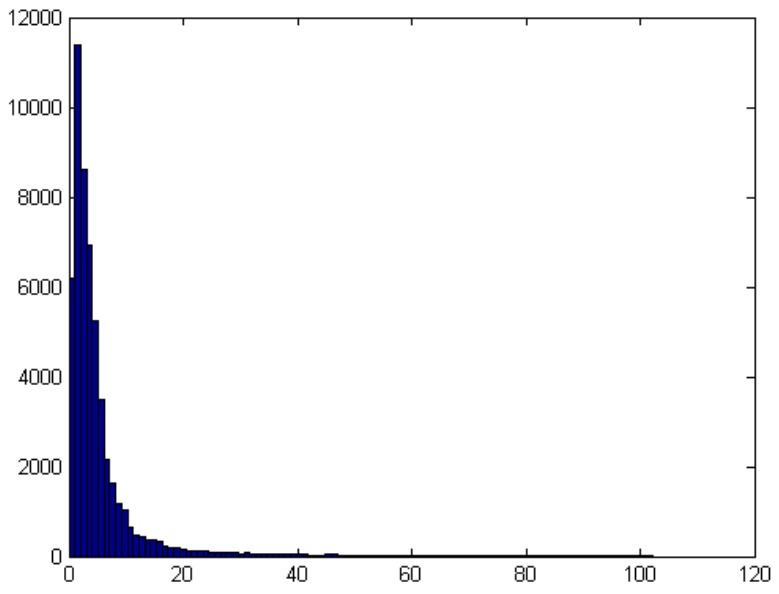

b. Segmented vs observer1 posterior ribs.

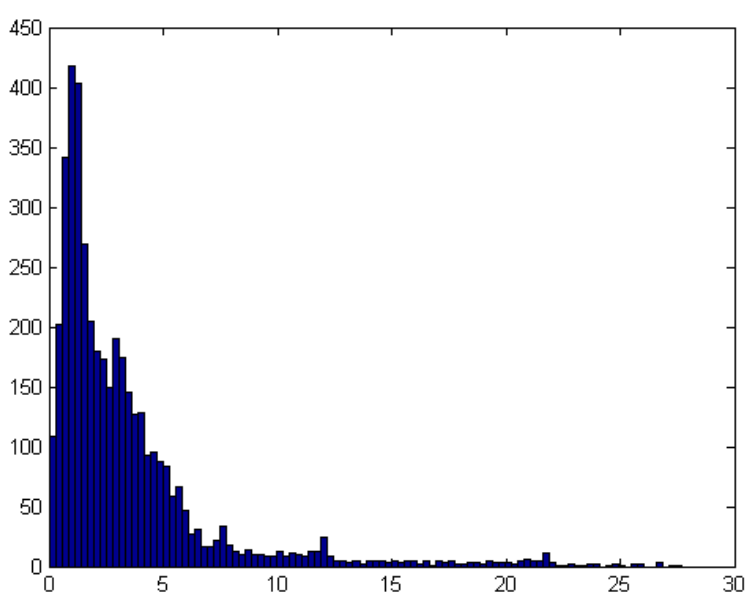

d.Inter-observer posterior ribs.

Fig. 5. Distribution of distances in pixel between the points of the reference and segmented rib borders restricted to the viewable lung field.

the bone shadow suppressed images on the lesion detector output but still excluding the three features based on bone outlines, while the third configuration both used the bone shadow free images and the new features. For each configuration we retrained the lesion detector and generated FROC curves using the methodology described before. The results can be seen in Figure 7

The improvement when using rib-shadow-free images is clear. At constant $63 \%$ sensitivity the number of false positives could be reduced from 2.94 to 1.4. Utilizing the new features, the number of false positives falls further to 1.23. This means $52 \%$ of false detections could be eliminated which is a great benefit for radiologists and physicians having to examine much less false CADe results at screening. Fixing the number of false detections to two yields sensitivity values of $57 \%, 66.7 \%$ and $68 \%$ for the unprocessed, the bone eliminated and the feature including configurations respectively. Finding $19 \%$ more lesions is a huge benefit in this case. Roughly the same improvement is valid for working points at lower false positive rates. Two ex-

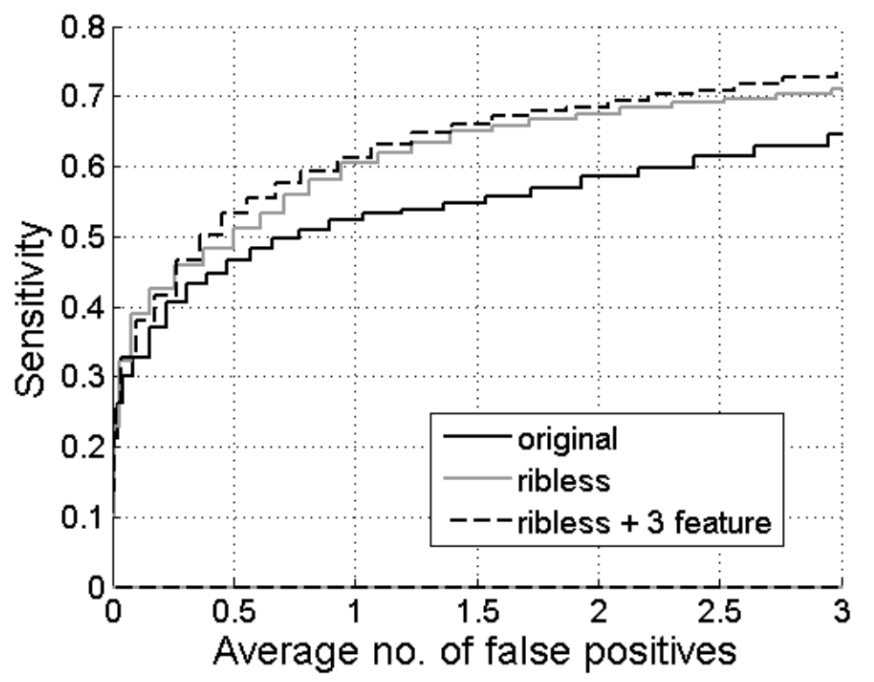

Fig. 7. FROC curves of the lesion detector system omitting bone shadow suppression (black line), the same system using preprocessed images without bone shadows (gray line) and the system utilizing both the three new features and preprocessed images (dashed line). The usage of cleared images greatly improves detection accuracy, while the new features show a slight extra benefit. 

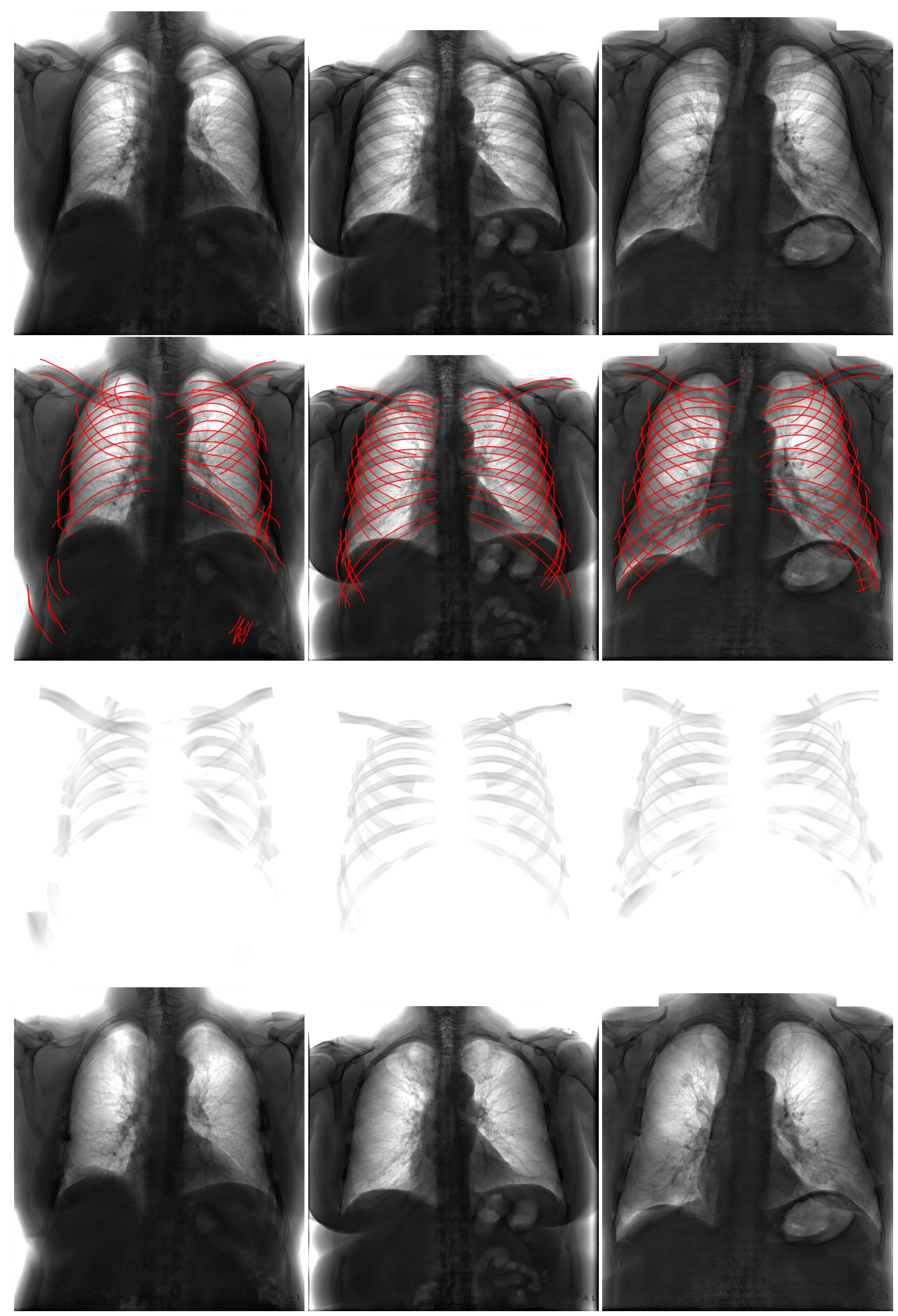

Fig. 6. The results of rib elimination. Original images (first row), result of bone segmentation (second row), calculated bone shadows (third row) and bone

eliminated images (fourth row). Left, center and right columns show the worst, the median and the best segmentation results respectively. 
ample changes can be seen in Figure 8 . In the first case a false negative changed into a true positive, while in the second case a false positive finding could be removed from the output. The majority of the changes were similar to these cases.

After seeing the benefits in detection accuracy we wanted to see how bone shadows and their removal affect the output distribution of the CADe system. We assumed that bone shadows and especially bone crossings cause many false positives for the original system and less or not at all for the new version. We conducted two tests to support our hypothesis.

In the first test we investigated the overlap of the false findings with bone shadows. We expected the false findings to overlap less often with bones after elimination. Moreover, in the ideal case when no bone shadows are present, the event of falsely marking an area as a lesion should be independent from the area being overlapped by a bone shadow. To test this independence we considered a simple pixel-wise model. Let $l_{i}$ be the indicator of marking the $\mathrm{i}^{\text {th }}$ pixel falsely as a lesion and $b_{i}$ the indicator of the $\mathrm{i}^{\text {th }}$ pixel belonging to a bone shadow. Let's assume that $l_{i}$-s for all $i$ are independent samples of the same indicator random variable $L$ and analogously $b_{i}$-s are generated from the indicator random variable $B^{1}$ The probability $P(B \mid L)$ should converge to $P(B)$ if we weaken the connection between the two variables and in the independent case they should be equal. Also the Pearson's correlation coefficient should be close to zero for weak dependency. $P(B \mid L)=P(B, L) / P(L)$ can be estimated as the overlap between false positive lesions and bone shadows over the entire area of false positives. $P(B)$ is simply the total area of bone shadows.

Table 7 shows the results of this test. The false positive area overlapping bones clearly decreases and approaches the total area of bone shadows as expected; however, we can still see a gap suggesting that some false positive findings are still caused by bone shadows. The Pearson's correlation coefficient gets closer to zero by an order of magnitude, although it was already small in the original case. Table 8 shows the same numbers for bone crossings yielding the same conclusion.

Tab. 7. Area fraction of false positive candidates overlapping with bone shadows.

\begin{tabular}{lccc}
\hline & Unprocessed & $\begin{array}{c}\text { Rib- } \\
\text { shadow-free }\end{array}$ & $\begin{array}{c}\text { Rib-shadow-free } \\
+3 \text { features }\end{array}$ \\
\hline Total bone area & 0.542 & 0.542 & 0.542 \\
FP area overl. bones & 0.791 & 0.625 & 0.584 \\
Pearson's correlation & 0.0413 & 0.014 & 0.0078 \\
\hline
\end{tabular}

In the second test we analyzed the independence of a candidate being false positive and its overlap with bone shadows as two random variables. We decided to use Pearson's chi-squared test to test independence, so we needed to discretize the vari-

\footnotetext{
${ }^{1}$ This model is obviously incorrect as neither independence nor the identity of distributions hold, but for a big enough sample it is a close enough estimate of reality for the purposes of this test.
}

Tab. 8. Area fraction of false positive candidates overlapping with shadows of bone crossings.

\begin{tabular}{lccc}
\hline & Unprocessed & $\begin{array}{c}\text { Rib- } \\
\text { shadow-free }\end{array}$ & $\begin{array}{c}\text { Rib-shadow-free } \\
+3 \text { features }\end{array}$ \\
\hline Total bone X area & 0.121 & 0.121 & 0.121 \\
FP area overl. bone X-s & 0.317 & 0.166 & 0.134 \\
Pearson's correlation & 0.05 & 0.0119 & 0.0038 \\
\hline
\end{tabular}

ables. The first one is already binary while the overlap feature was quantized to three bins: full overlap, partial overlap and no overlap with a $5 \%$ tolerance. We demonstrated the resulting p-values in Table 9. We ran the tests for the same three configurations as above. Using a predefined significance level of $1 \%$ the first two cases are clearly significant meaning that false positiveness depends on - or at least correlates with - the overlap with bones while in the third case we can no longer be sure that the relation exists. However, we should note that a $\mathrm{p}$-value of 0.049 suggests further investigation on a larger sample. We ran the same test using the bone crossing overlap as the second variable. Here, using the original system shows a clear correlation, while the results for the versions exploiting bone information are insignificant. These results do not contradict with our original hypothesis; however, the proof of independence needs more studies.

\section{Conclusion and future work}

To summarize our work, we developed a CADe system capable of automatically detecting various lung lesions. In our solution the ribcage and clavicle shadows are removed before lesion detection. The cleaned images are analyzed by three different filters for lesions and a hierarchy of SVM classifiers is used to reduce the number of false findings.

We evaluated the bone shadow segmentation algorithm and found that it can detect approximately $80 \%$ of bone shadows with false findings around $10 \%$. For the more disturbing posterior part it can detect as much as $84 \%$. According to our interobserver tests, we cannot really hope for more than $94 \%$ sensitivity. When a bone is identified correctly, the segmentation border is accurate within $1 \mathrm{~mm}$. This is again close to the approximated lower bound determined by the inter observer analysis.

Our measurements showed that lesion detection performance can be greatly improved by removing bone shadows and also a bit more by using the bone segmentation information as classifier input. The former reduced the number of false findings by $52 \%$ from 2.94 to 1.4 at $63 \%$ sensitivity, while the latter resulted in a further decrease of $12 \%$ to 1.23 false positives per image.

We also tested if the remaining false findings are still dependent on the overlap with bone shadows. The results confirmed that dependence is much weaker on the processed images, although we could not prove complete independence. This means that some remainders of bone shadows are still causing a few 
Fig. 8. Samples of detector output before (left) and after (right) bone shadow suppression. The first example (top) is a true nodule while the second (bottom) is a healthy region.

Tab. 9. Resulting p-values of independence tests between bone overlapping and false positiveness.

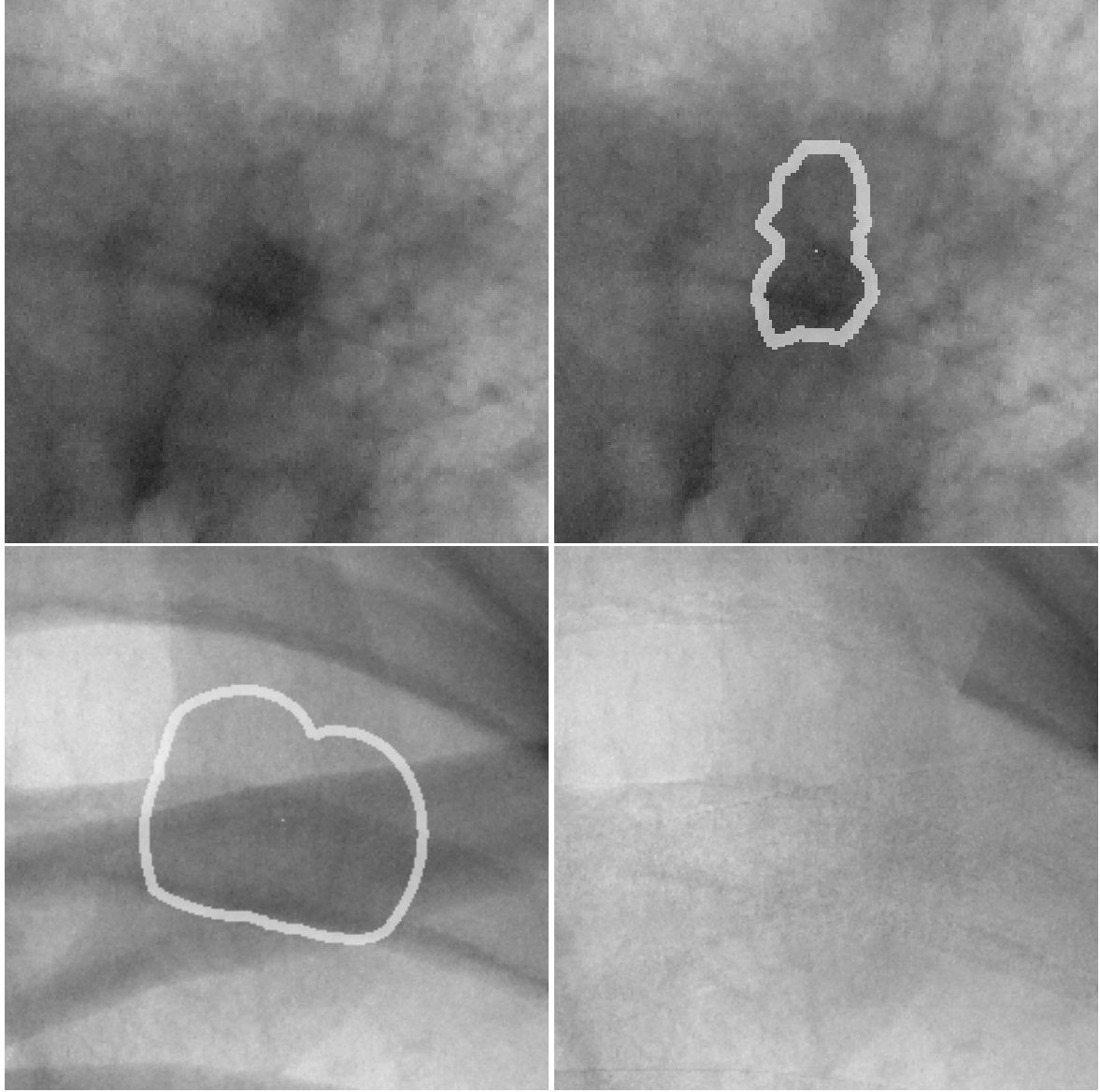

\begin{tabular}{lccc}
\hline & Original image & Rib-shadow-free image & $\begin{array}{c}\text { Rib-free } \\
+3 \text { features }\end{array}$ \\
\hline Overlap w. bones & 0.0001 & 0.0002 & 0.049 \\
Overlap w. bone X-s & 0.0015 & 0.61 & 0.2 \\
\hline
\end{tabular}

false findings, but improving the accuracy of bone shadow removal is not likely to result in further drastic improvements.

While evaluating our study we identified directions for further investigation. As some of the lung lesions are under the shadow of the heart and below the diaphragm it is a straightforward idea to extend the search for lesions to these parts of the image. For this we would need to balance contrast in these areas. Fortunately the bone removal algorithm seems to be capable for this task with only small modifications. Extending the search can hopefully lead to an increase in sensitivity.

Overall we believe our study contributes to the CADe community by showing the utility of bone shadow and more generally anatomic noise removal for the purposes of automatic lung lesion detection. Although a few other research groups are also putting effort in this approach of image cleaning, a comprehensive performance comparison for automatic lesion detection has not been made so far. We also believe our method for segmenting the bone shadows is performing at least as good as alternative solutions due to its high accuracy and the inclusion of the anterior rib segments.

\section{References}

1 Kramer BSaB Christine D. and Aberle, Lung cancer screening with low-dose helical CT: results from the National Lung Screening Trial (NLST), Journal of Medical Screening, 18(3), (2011), 109-111, DOI 10.1258/jms.2011.011055.

2 Doi K, Computer-aided diagnosis in medical imaging: historical review, current status and future potential, Computerized Medical Imaging and Graphics, 31(4-5), (2007), 198-211.

3 Kobayashi T, Xu X-W, MacMahon H, Metz C, Doi K, Effectof a computeraided diagnosis scheme on radiologists' performancein detection of lung nodules on radiographs, Radiology, 199, (1996), 843-848.

4 MacMahon H, Engelmann R, Behlen FM, Hoffmann KR, Ishida T, Roe C, Metz CE, Doi K., Computer-aided Diagnosis of Pulmonary Nodules: Results of a Large-Scale Observer Test, Radiology, 213, (1999), 723-726.

\section{De Boo DW, Prokop M, Uffmann M, Van Ginneken B, Schaefer-} Prokop C, Computer-aided detection (CAD) of lung nodules and small tumours on chest radiographs, European Journal of Radiology, 72(2), (2009), 218-225.

6 Chen S, Suzuki K, MacMahon H, Development and evaluation of a computer-aided diagnostic scheme for lung nodule detection in chest radiographs by means of two-stage nodule enhancement with support vector classification, Medical Physics, 38, (2011), 1844.

7 Snoeren PR, Litjens GJS, van Ginneken B, Karssemeijer N, Training a Computer Aided Detection System with Simulated Lung Nodules in Chest Radiographs. 
8 Hardie RC, Rogers SK, Wilson T, Rogers A, Performance analysis of a new computer aided detection system for identifying lung nodules on chest radiographs, Medical Image Analysis, 12(3), (2008), 240-258.

9 Campadelli P, Casiraghi E, Artioli D, A fully automated method for lung nodule detection from postero-anterior chest radiographs, Medical Imaging, IEEE Transactions on, 25(12), (2006), 1588-1603.

10 Schilham AMR, Van Ginneken B, Loog M, A computer-aided diagnosis system for detection of lung nodules in chest radiographs with an evaluation on a public database, Medical Image Analysis, 10(2), (2006), 247-258.

11 Coppini G, Diciotti S, Falchini M, Villari N, Valli G, Neural networks for computer-aided diagnosis: detection of lung nodules in chest radiograms, Information Technology in Biomedicine, IEEE Transactions on, 7(4), (2003), 344-357.

12 Wei J, Hagihara Y, Shimizu A, Kobatake H, Optimal image feature set for detecting lung nodules on chest X-ray images, Proc. of CARS2002, (2002), 706-711.

13 Orbán G, Horváth G, Algorithm fusion to improve detection of lung cancer on chest radiographs, International Journal of Intelligent Computing and Cybernetics, 5(1), (2012), 111-144.

14 Brody WR, Butt G, Hall A, Macovski A, A method for selective tissue and bone visualization using dual energy scanned projection radiography, Medical physics, 8, (1981), 353.

15 Suzuki K, Abe $\mathbf{H}$, MacMahon $\mathbf{H}$, et al., Image-processing technique for suppressing ribs in chest radiographs by means of massive training artificial neural network (MTANN), Medical Imaging, IEEE Transactions on, 25(4), (2006), 406-416.

16 Loog M, van Ginneken B, Schilham A, Filter learning: application to suppression of bony structures from chest radiographs, Medical image analysis, 10(6), (2006), 826-840.

17 Rasheed T, Ahmed B, Khan M, Bettayeb M, Lee S, Kim T-S, Rib suppression in frontal chest radiographs: A blind source separation approach, In: Signal Processing and Its Applications, 2007. ISSPA 2007. 9th International Symposium on, IEEE, 2007, pp. 1-4.

18 Toriwaki Jl, Suenaga Y, Negoro T, Fukumura T, Pattern recognition of chest $X$-ray images, Computer Graphics and Image Processing, 2(3), (1973), 252-271.

19 Wechsler H, Sklansky J, Finding the rib cage in chest radiographs, Pattern Recognition, 9(1), (1977), 21-30.

20 de Souza $\mathbf{P}$, Automatic rib detection in chest radiographs, Computer vision, graphics, and image processing, 23(2), (1983), 129-161.

21 Yue Z, Goshtasby A, Ackerman LV, Automatic detection of rib borders in chest radiographs, Medical Imaging, IEEE Transactions on, 14(3), (1995), 525-536.

22 van Ginneken B, ter Haar Romeny BM, Automatic delineation of ribs in frontal chest radiographs, In: Proceedings of SPIE, Vol. 3979, 2000, p. 825.

23 Sakaida H, Oosawa A, Shimura K, Rib shape recognition in lung $x$-ray images for intelligent assistance, FUJI FILM RESEARCH AND DEVELOPMENT, 52, (2007), 99.

24 Van Ginneken B, Stegmann MB, Loog M, Segmentation of anatomical structures in chest radiographs using supervised methods: a comparative study on a public database, Medical Image Analysis, 10(1), (2006), 19-40.

25 Vogelsang F, Weiler F, Dahmen J, Kilbinger M, Wein B, Günther R, Detection and compensation of rib structures in chest radiographs for diagnose assistance, In: Proceedings of the International Symposium on Medical Imaging, Vol. 3338, Citeseer, 1998, p. 1.

26 Park M, Jin JS, Wilson LS, Detection of abnormal texture in chest X-rays with reduction of ribs, In: Proceedings of the Pan-Sydney area workshop on Visual information processing, Australian Computer Society, Inc., 2004, pp. 71-74.

27 Simkó G, Orbán G, Máday P, Horváth G, Elimination of clavicle shadows to help automatic lung nodule detection on chest radiographs, In: 4th Euro- pean Conference of the International Federation for Medical and Biological Engineering, Springer, 2009, pp. 488-491.

28 Freedman MT, Lo S-CB, Seibel JC, Bromley CM, Lung nodules: improved detection with software that suppresses the rib and clavicle on chest radiographs, Radiology, 260(1), (2011), 265-273.

29 Soleymanpour E, Pourreza HR, et al., Fully Automatic Lung Segmentation and Rib Suppression Methods to Improve Nodule Detection in Chest Radiographs, Journal of Medical Signals and Sensors, 1(3), (2011), 191.

30 Li F, Hara T, Shiraishi J, Engelmann R, MacMahon H, Doi K, Improved Detection of Subtle Lung Nodules by Use of Chest Radiographs With Bone Suppression Imaging: Receiver Operating Characteristic Analysis With and Without Localization, American Journal of Roentgenology, 196(5), (2011), W535-W541.

31 Novak RD, Novak NJ, Gilkeson R, Mansoori B, Aandal GE, A Comparison of Computer-Aided Detection (CAD) Effectiveness in Pulmonary Nodule Identification Using Different Methods of Bone Suppression in Chest Radiographs, Journal of Digital Imaging, (2013), 1-6.

32 Yan Z, Zhang J, Zhang S, Metaxas DN, Automatic Rapid Segmentation of Human Lung from $2 D$ Chest $X$-Ray Images.

33 Horváth Á, Horvath G, Segmentation of Chest X-ray Radiographs, a New Robust Solution, 5th European Conference of the International Federation for Medical and Biological Engineering, (2012), 655-658.

34 Horváth Á, Locating clavicles on chest radiographs, In: Proceedings of the 19th PhD Minisymposium, Budapest University of Technology and Economics, Department of Measurement and Information Systems, 2012, pp. 62-63.

35 Juhász S, Horváth Á, Nikházy L, Horváth G, Horváth Á, Segmentation of Anatomical Structures on Chest Radiographs, In: Magjarevic RaB Panagiotis D. and Pallikarakis (ed.), XII Mediterranean Conference on Medical and Biological Engineering and Computing 2010, IFMBE Proceedings, Vol. 29, Springer Berlin Heidelberg, 2010, pp. 359-362, DOI 10.1007/978-3642-13039-7_90.

36 Horváth Á, Statistical Approach Towards Ribcage Segmentation, In: Proceedings of the 20th PhD Minisymposium, Budapest University of Technology and Economics, Department of Measurement and Information Systems, 2013, pp. 26-29.

37 Pereira C, Mendonça A, Campilho A, Evaluation of Contrast Enhancement Filters for Lung Nodule Detection, In: ICIAR 2007 Proceedings, Vol. 1; Montreal, Canada, 2007, pp. 878-888.

38 Lee JS, Digital image enhancement and noise filtering by use of local statistics, Pattern Analysis and Machine Intelligence, IEEE Transactions on, 2, (1980), 165-168.

39 Cortes C, Vapnik V, Support-vector networks, Machine learning, 20(3), (1995), 273-297.

40 Heath MD, Bowyer KW, Mass detection by relative image intensity, In: Proceedings of the 5 th International Workshop on Digital Mammography (IWDM-2000), 2000, pp. 219-225.

41 Haralick RM, Shanmugam K, Dinstein IH, Textural features for image classification, IEEE Transactions on systems, man and cybernetics, 3(6), (1973), 610-621. 